\title{
FINITELY-VALUED COHOMOLOGY GROUPS
}

\author{
JOHN W. KEESEE ${ }^{1}$
}

In this note it will be shown that finitely-valued functions are adequate to determine the Alexander-Kolmogoroff cohomology groups of a compact Hausdorff space. This is effected by exhibiting a homomorphism of the finitely-valued groups into the general groups which is an isomorphism onto if the space is compact. We assume familiarity with Spanier's [2] ${ }^{2}$ development of the AlexanderKolmogoroff groups although the definitions are repeated here.

Let $X$ be an arbitrary topological space and $G$ a fixed abelian group. Denote by $\Phi^{p}(X)$ the group of all functions ( $p$-functions) from $X^{p+1}$ (the Cartesian product of $X$ with itself $p+1$ times) to $G$. Let $\left\{N\left(\Delta X^{p+1}\right)\right\}$ represent the open sets containing the diagonal of $X^{p+1}$. Then $\Phi_{0}^{p}(X)$ is the subgroup of $\Phi^{p}(X)$ consisting of those $p$ functions which are zero on some $N\left(\Delta X^{p+1}\right)$. There is a homomorphism $\bar{\delta}$ of $\Phi^{p}(X)$ into $\Phi^{p+1}(X)$ defined by the formula

$$
\bar{\delta} \phi\left(x_{0}, x_{1}, \cdots, x_{p+1}\right)=\sum_{i=0}^{p+1}(-1)^{i} \phi\left(x_{0}, x_{1}, \cdots, x_{i-1}, x_{i+1}, \cdots, x_{p+1}\right) .
$$

This homomorphism has the property that $\bar{\delta} \bar{\delta}=0$. The subgroup $\Phi_{Z}^{p}(X)=\bar{\delta}^{-1} \Phi_{0}^{p+1}(X)$ is called the group of $p$-cocycles, and $\Phi_{B}^{p}(X)$, the group of $p$-coboundaries, is defined to be $\delta \Phi^{p-1}(X)+\Phi_{0}^{p}(X)$. The $p$ th cohomology group of the space $X$, denoted by $H^{p}(X)$, is the quotient group $\Phi_{Z}^{p}(X) / \Phi_{B}^{p}(X)$.

For each $f: X \rightarrow Y$ ( $f$ not necessarily continuous) there is defined a homomorphism $f^{\#}: \Phi^{p}(Y) \rightarrow \Phi^{p}(X)$ by

$$
f^{\sharp} \phi\left(x_{0}, x_{1}, \cdots, x_{p}\right)=\phi\left(f\left(x_{0}\right), f\left(x_{1}\right), \cdots, f\left(x_{p}\right)\right), \quad \phi \in \Phi^{p}(Y) .
$$

If $f$ is a map (= continuous function), $f^{*}$ carries each of the subgroups $\Phi_{0}^{p}(Y), \Phi_{B}^{p}(Y)$, and $\Phi_{Z}^{p}(Y)$ into the corresponding subgroup of $\Phi^{p}(X)$.

Let $A$ be a subset of $X$ and $i: A \rightarrow X$ the identity map. Then

$$
\begin{aligned}
& \Phi^{p}(X, A)=i^{t-1} \Phi_{0}^{p}(A), \quad \Phi_{0}^{p}(X, A)=\Phi_{0}^{p}(X), \\
& \Phi_{B}^{p}(X, A)=\bar{\delta} \Phi^{p-1}(X, A)+\Phi_{0}^{p}(X, A), \\
& \Phi_{Z}^{p}(X, A)=\Phi^{p}(X, A) \cap \Phi_{Z}^{p}(X) .
\end{aligned}
$$

Presented to the Society, April 2, 1949; received by the editors June 3, 1949.

1 This work was done under Contract N7-onr-534, Task Order III, Navy Department, Office of Naval Research.

2 Numbers in brackets refer to the references cited at the end of the paper. 
The pth cohomology group of $X \bmod A$ is $H^{p}(X, A)$ $=\Phi_{Z}^{p}(X, A) / \Phi_{B}^{p}(X, A)$.

If $f$ is a function on $X$ to $Y$ that carries a subset $A$ of $X$ into a subset $B$ of $Y, f$ is said to be on $(X, A)$ to $(Y, B)$. We write

$$
f: \quad(X, A) \rightarrow(Y, B) .
$$

If $f:(X, A) \rightarrow(Y, B)$ is a map, $f^{t}$ carries $\Phi^{p}(Y, B), \Phi_{Z}^{p}(Y, B)$, and $\Phi_{B}^{p}(Y, B)$ into $\Phi^{p}(X, A), \Phi_{Z}^{p}(X, A)$, and $\Phi_{B}^{p}(X, A)$ respectively.

For a fixed pair of functions $f$ and $g$ on $(X, A)$ to $(Y, B)$ there is a function $D: \Phi^{p+1}(Y, B) \rightarrow \Phi^{p}(X, A)$ defined by $D \phi\left(x_{0}, x_{1}, \cdots, x_{p}\right)$ $=\sum_{i=0}^{p}(-1)^{i} \phi\left(g\left(x_{0}\right), g\left(x_{1}\right), \cdots, g\left(x_{i}\right), f\left(x_{i}\right), \cdots, f\left(x_{p}\right)\right)$. Then for $p>0$ and $\phi \in \Phi^{p}(Y, B), f^{\ddagger} \phi-g^{*} \phi=\bar{\delta} D \phi+D \bar{\delta} \phi$. If $p=0, f^{\sharp} \phi-g^{\dagger} \phi$ $=D \bar{\delta} \phi$.

The purpose of this paper is to consider the groups $H_{F}^{p}(X, A)$ defined as follows. Let $F^{p}(X)$ be the subgroup of $\Phi^{p}(X)$ consisting of those $p$-functions which have only a finite number of elements of $G$ as values. Define $F^{p}(X, A)=\Phi^{p}(X, A) \cap F^{p}(X), F_{0}^{p}(X, A)=\Phi_{0}^{p}(X, A)$ $\cap F^{p}(X), F_{B}^{p}(X, A)=\bar{\delta} F^{p-1}(X, A)+F_{0}^{p}(X, A)$ (when $p=0, F_{B}^{p}(X, A)$ $=0), F_{Z}^{p}(X, A)=\Phi_{Z}^{p}(X, A) \cap F^{p}(X)$. Then $H_{F}^{p}(X, A)$ is the quotient group $F_{Z}^{p}(X, A) / F_{B}^{p}(X, A)$. It will be shown that for an arbitrary space $X$, there is a homomorphism $\tau: H_{P}^{p}(X, A) \rightarrow H^{p}(X, A)$ which is an isomorphism onto if $X$ is a compact Hausdorff space and $A$ is closed.

Lemma 1. Let $M$ and $N$ be abelian groups with subgroups $M_{0}$ and $N_{0}$ and $\lambda$ a homomorphism of $M$ into $N$ such that $\lambda\left(M_{0}\right) \subset N_{0}$. Let $\alpha: M \rightarrow M / M_{0}$ and $\beta: N \rightarrow N / N_{0}$ be the natural homomorphisms. Then there is a homomorphism $\rho: M / M_{0} \rightarrow N / N_{0}$ defined by $\rho \alpha(x)=\beta \lambda(x)$ for $x \in M$. Furthermore, the kernel of $\rho$ is $\lambda^{-1}\left(N_{0}\right) / M_{0}$ and the image of $M / M_{0}$ under $\rho$ is $\left(\lambda(M)+N_{0}\right) / N_{0}$.

The method of proof is evident.

The results of Lemma 1 are used to define the homomorphism $\tau$ as follows: Denote by $\gamma: \Phi_{Z}^{p}(X, A) \rightarrow H^{p}(X, A)$ and $\nu: F_{Z}^{p}(X, A)$ $\rightarrow H_{F}^{p}(X, A)$ the natural homomorphisms and let $\sigma: F_{Z}^{p}(X, A)$ $\rightarrow \Phi_{Z}^{p}(X, A)$ be the identity homomorphism. Define $\tau: H_{F}^{p}(X, A)$ $\rightarrow H^{p}(X, A)$ by $\tau \nu(\phi)=\gamma \sigma(\phi)$ for $\phi \in F_{Z}^{p}(X, A)$. It follows that $\tau$ is a homomorphism, the kernel of $\tau$ is $\Phi_{B}^{p}(X, A) \cap F^{p}(X) / F_{B}^{p}(X, A)$, and the image of $H_{F}^{p}(X, A)$ under $\tau$ is $F_{Z}^{p}(X, A)+\Phi_{B}^{p}(X, A) / \Phi_{B}^{p}(X, A)$.

The next two lemmas are concerned with properties of subsets of $G$ that satisfy the following two conditions: (1) If a subset $H$ of $G$ has property $\mathrm{M}$, then $(-H)$ has $\mathrm{M}$. (2) If $H_{1}$ and $H_{2}$ have $\mathrm{M}$, then $\left(H_{1}+H_{2}\right)$ has $\mathrm{M}$. For such a property $\mathrm{M}$, define $M^{p}(X, A)$ to be all 
$\phi \in \Phi^{p}(X, A)$ such that $\phi$ carries some $N\left(\Delta X^{p+1}\right)$ into a set $H$ with property $M$.

In what follows, if $U$ is a covering of $X, N_{p}(U)$ will denote the set $U\left[U^{p} \mid U \in V\right]$.

Lemma 2. Hypothesis: (a) $X$ and $Y$ are topological spaces, $f$ and $g$ are functions on $(X, A)$ to $(Y, B)$;

(b) $\phi \in \Phi^{p}(Y, B), \bar{\delta} \phi \in M^{p+1}(Y, B)$;

(c) $\mathcal{V}$ is a covering of $Y$ such that $\phi=0$ on $N_{p+1}(\mathcal{V}) \cap B^{p+1}$, and $\bar{\delta} \phi$ carries $N_{p+2}(\mathcal{V})$ into a subset $N$ of $G$ with property $\mathrm{M}$;

(d) $U$ is a covering of $X$ such that for each $U \in U$, there is a $V \in V$ for which $f(U) \cup g(U) \subset V$.

Conclusion: For $p>0, f^{\dagger} \phi-g^{\dagger} \phi \in \bar{\delta} \Phi^{p-1}(X, A)+M^{p}(X, A)$; for $p=0, f^{\sharp} \phi-g^{\sharp} \phi \in M^{p}(X, A)$.

Proof. It is sufficient to show (i) if $p \geqq 0, D \bar{\delta} \phi \in M^{p}(X, A)$ and(ii) if $p>0, \bar{\delta} D \phi \in \bar{\delta} \Phi^{p-1}(X, A)$. First consider $\left(x_{0}, \cdots, x_{p}\right) \in N_{p+1}(U)$ $\cap A^{p+1}$. Then $D \bar{\delta} \phi\left(x_{0}, \cdots, x_{p}\right)=\sum_{i=0}^{p}(-1)^{i}(\bar{\delta} \phi)\left(g\left(x_{0}\right), \cdots, g\left(x_{i}\right)\right.$, $\left.f\left(x_{i}\right), \cdots, f\left(x_{p}\right)\right)$. But $\left(g\left(x_{0}\right), \cdots, g\left(x_{i}\right), f\left(x_{i}\right), \cdots, f\left(x_{p}\right)\right) \in N_{p+2}(\mathcal{V})$ $\cap B^{p+2}$ so $D \bar{\delta} \phi=0$ on $N_{p+1}(U) \cap A^{p+1}$ and therefore $D \bar{\delta} \phi \in \Phi^{p}(X, A)$. Also for $\left(x_{0}, \cdots, x_{p}\right) \in N_{p+1}(U), D \bar{\delta} \phi\left(x_{0}, \cdots, x_{p}\right) \in \sum_{i=0}^{p}(-1)^{i} N$. But this set has property $\mathrm{M}$ since $N$ has and $D \bar{\delta} \phi \in M^{p}(X, A)$. Second, for $p>0$, take $\left(x_{0}, \cdots, x_{p-1}\right) \in N_{p}(U) \cap A^{p}$. Then $\left(g\left(x_{0}\right)\right.$, $\left.\cdots, g\left(x_{i}\right), f\left(x_{i}\right), \cdots, f\left(x_{p-1}\right)\right) \in N_{p+1}(\mathcal{V}) \cap B^{p+1}$ and $D \phi\left(x_{0}, \cdots\right.$, $\left.x_{p-1}\right)=0$. So $D \phi \in \Phi^{p-1}(X, A)$. This completes the proof of the lemma.

Lemma 3. If $X$ is a compact Hausdorff space and $A$ is closed, then $\Phi^{p}(X, A) \cap \bar{\delta}^{-1} M^{p+1}(X, A) \subset \bar{\delta} \Phi^{p-1}(X, A)+M^{p}(X, A)+F^{p}(X, A)$.

Proof. If $\phi \in \Phi^{p}(X, A) \cap \bar{\delta}^{-1} M^{p+1}(X, A)$, then there exists a covering $\mathcal{V}$ of $X$ for which $\phi=0$ on $N_{p+1}(\mathcal{V}) \cap A^{p+1}$ and $\bar{\delta} \phi$ carries $N_{p+2}(\mathcal{V})$ into a subset of $G$ with property M. Let $U$ be a finite star refinement of $\mathcal{V}\left[1\right.$, p. 324]. Write $V=\left[U_{1}, U_{2}, \cdots, U_{n}\right]$. To each $U_{j}$ for which $U_{j} \cap A \neq \square$, assign $\bar{x}_{j} \in U_{j} \cap A$. To each $U_{j}$ such that $U_{j} \cap A=\square$, assign $\bar{x}_{j} \in U_{j}$. Define $f:(X, A) \rightarrow(X, A)$ by $f(x)=\bar{x}_{j}$ where $j$ is the least integer for which $x \in U_{j}$. Then $f(X)$ is a finite subset of $X$ and $f^{\dagger} \phi \in F^{p}(X)$. Also $f^{\dagger} \phi \in F^{p}(X, A)$, for consider $\left(x_{0}, \cdots, x_{p}\right) \in N_{p+1}(U) \cap A^{p+1}$. Then $x_{i} \in U_{k}(i=0, \cdots, p)$ and there exists $V_{0} \in \mathcal{V}$ such that $V_{0} \supset$ the star of $U_{k}$. Then $f\left(U_{k}\right) \subset$ the star of $U_{k}$ implies $f\left(x_{i}\right) \in$ the star of $U_{k}$. Hence $\left(f\left(x_{0}\right), f\left(x_{1}\right), \cdots\right.$, $\left.f\left(x_{p}\right)\right) \in V_{0}^{p+1}$, and $f^{\sharp} \phi\left(x_{0}, \cdots, x_{p}\right)=\phi\left(f\left(x_{0}\right), \cdots, f\left(x_{p}\right)\right)=0$. Therefore $f^{\star} \phi \in \Phi^{p}(X, A)$. Let $i:(X, A) \rightarrow(X, A)$ be the identity function; then for each $U \in V$, there exists $V \in \mathcal{V}$ such that $i(U) \cup f(U) \subset V$. Now by Lemma $2, i^{f} \phi-f^{\ddagger} \phi \in \bar{\delta} \Phi^{p-1}(X, A)+M^{p}(X, A)$ or 


$$
\phi \in \bar{\delta} \Phi^{p-1}(X, A)+M^{p}(X, A)+F^{p}(X, A) .
$$

For the next lemma define $L^{p}(X)$ to be the group of $p$-functions which assume only a finite number of values on some $N\left(\Delta X^{p+1}\right)$, and let $L^{p}(X, A)=L^{p}(X) \cap \Phi^{p}(X, A)$. It follows that $L^{p}(X, A)=F^{p}(X, A)$ $+\Phi_{0}^{p}(X, A)$ and $\bar{\delta} L^{p}(X, A) \subset \bar{\delta} F^{p}(X, A)+\Phi_{0}^{p+1}(X, A)$.

LemMa 4. If $X$ is a compact Hausdorff space and $A$ is closed, then $\Phi^{p}(X, A) \cap \bar{\delta}^{-1} L^{p+1}(X, A) \subset \Phi_{B}^{p}(X, A)+F^{p}(X, A)$.

Proof. Since the property of being finite is a property of the type of Lemma 2, it follows by Lemma 3 that

$$
\Phi^{p}(X, A) \cap \bar{\delta}^{-1} L^{p+1}(X, A) \subset \bar{\delta} \Phi^{p-1}(X, A)+L^{p}(X, A) .
$$

But $\bar{\delta} \Phi^{p-1}(X, A)+L^{p}(X, A)=\bar{\delta} \Phi^{p-1}(X, A)+\Phi_{0}^{p}(X, A)+F^{p}(X, A)$ $=\Phi_{B}^{p}(X, A)+F^{p}(X, A)$.

In the proof of the following theorem, repeated use is made of the so-called Dedekind property of groups, that is, if $A, B$, and $C$ are subgroups of a group $G$, and $A$ is contained in $C$, then

$$
(A+B) \cap C=(A \cap C)+(B \cap C) \text {. }
$$

Theorem. If $X$ is a compact Hausdorff space and $A$ is closed, then $\tau$ is an isomorphism onto.

Proof. (a) Show $\tau$ is an isomorphism. The kernel of $\tau$ is $\Phi_{B}^{p}(X, A)$ $\cap F^{p}(X) / F_{B}^{p}(X, A)$.

Now

$$
\begin{aligned}
\Phi_{B}^{p}(X, A) \cap F^{p}(X) & =\left[\bar{\delta} \Phi^{p-1}(X, A)+\Phi_{0}^{p}(X, A)\right] \cap F^{p}(X) \\
& =\left[\bar{\delta} \Phi^{p-1}(X, A)+\Phi_{0}^{p}(X, A)\right] \cap L^{p}(X) \cap F^{p}(X) \\
& =\left[\bar{\delta} \Phi^{p-1}(X, A) \cap L^{p}(X, A)+\Phi_{0}^{p}(X, A)\right] \cap F^{p}(X) .
\end{aligned}
$$

By Lemma $4 \bar{\delta} \Phi^{p-1}(X, A) \cap L^{p}(X, A) \subset \bar{\delta} \Phi_{B}^{p-1}(X, A)+\bar{\delta} F^{p-1}(X, A)$. Therefore, $\Phi_{B}^{p}(X, A) \cap F^{p}(X) \subset\left[\bar{\delta} F^{p-1}(X, A)+\Phi_{0}^{p}(X, A)\right] \cap F^{p}(X)$. But $\left[\bar{\delta} F^{p-1}(X, A)+\Phi_{0}^{p}(X, A)\right] \cap F^{p}(X)=\bar{\delta} F^{p-1}(X, A)+\Phi_{0}^{p}(X, A)$ $\cap F^{p}(X)=F_{B}^{p}(X, A)$. This shows that the kernel of $\tau$ is 0 .

(b) Show $\tau$ is onto. The image of $H_{P}^{p}(X, A)$ under $\tau$ is $F_{Z}^{p}(X, A)$ $+\Phi_{B}^{p}(X, A) / \Phi_{B}^{p}(X, A)$. In Lemma 3 define " $H$ has $\mathrm{M}$ " to mean “ $H=0$." Then $M^{p+1}(X, A)=\Phi_{0}^{p+1}(X, A)$ and

$$
\begin{aligned}
\Phi_{Z}^{p}(X, A) & \subset \bar{\delta} \Phi^{p-1}(X, A)+\Phi_{0}^{p}(X, A)+F^{p}(X, A) \\
& =\Phi_{B}^{p}(X, A)+F^{p}(X, A) .
\end{aligned}
$$

But since $\Phi_{B}^{p}(X, A) \subset \Phi_{Z}^{p}(X, A)$, it follows that $\Phi_{Z}^{p}(X, A) \subset \Phi_{B}^{p}(X, A)$ 
$+F_{Z}^{p}(X, A)$. The reverse inclusion is clear and the equality shows that $\tau$ is onto.

\section{REFERENCES}

1. S. Lefschetz, Algebraic topology, Amer. Math. Soc. Colloquium Publications, vol. 27, New York, 1942.

2. E. H. Spanier, Cohomology theory for general spaces, Ann. of Math. vol. 49 (1948) pp. 405-427.

TULANE UNIVERSITY

\section{A NOTE ON A COMBINATORIAL PROBLEM}

\section{H. J. RYSER}

The purpose of this note is to prove the following theorem.

TheOREM. Let $x_{1}, x_{2}, \cdots, x_{v}$ denote $v$ distinct elements and let $T_{1}, T_{2}, \cdots, T_{v}$ denote $v$ sets formed from these elements. Suppose further that each $T_{i}$ consists of exactly $k$ distinct elements and that every pair of distinct sets $T_{i}$ and $T_{j}$ have exactly $\lambda$ elements in common $(0<\lambda<k<v)$. Then

$$
\lambda=\frac{k(k-1)}{v-1} .
$$

The combinatorial problem under consideration makes its appearance in the study of projective planes, Hadamard matrices, and block designs. In the combinatorial problem of Todd arising in the study of Hadamard matrices it is usually assumed that $\lambda$ $=k(k-1) /(v-1)[3 ; 4] .{ }^{1}$ For the symmetrical block designs each element of the arrangement is required to occur exactly $k$ times, and it is then easy to verify that $\lambda=k(k-1) /(v-1)[1]$. Further theorems concerning the possibility of this combinatorial problem for a given $v, k$, and $\lambda$ may be found in [3].

To prove the theorem let the elements $x_{1}, \cdots, x_{v}$ be listed in a row, and let the sets $T_{1}, \cdots, T_{v}$ be listed in a column. Form the incidence matrix $A$ of the arrangement in the usual way by inserting a one in column $i$ and row $j$ if $x_{i}$ belongs to set $T_{j}$, and a zero in the contrary case $[2 ; 3]$. Now let $s_{i}$ denote the sum of column $i$ of the

Presented to the Society, September 2, 1949; received by the editors June 9, 1949.

${ }^{1}$ Numbers in brackets refer to the references cited at the end of the paper. 